\title{
The flavivirus dengue induces hypertrophy of white matter astrocytes
}

\author{
Kim M. Lee ${ }^{1,2} \cdot$ Kevin B. Chiu ${ }^{2,3}$ - Hope A. Sansing ${ }^{2}$ - Peter J. Didier ${ }^{2}$. \\ Andrew A. Lackner ${ }^{1,2,4}$ • Andrew G. MacLean ${ }^{1,2,4}$
}

Received: 10 March 2016 / Revised: 9 May 2016 / Accepted: 26 May 2016 / Published online: 6 June 2016

(C) Journal of NeuroVirology, Inc. 2016

\begin{abstract}
Flaviviruses, including Zika and dengue (DENV), pose a serious global threat to human health. Of the $50+$ million humans infected with DENV annually, approximately 1$3 \%$ progress to severe disease manifestations, dengue hemorrhagic fever (DHF) or dengue shock syndrome (DSS). Several factors are suspected to mediate the course of infection and pathogenesis of DENV infection. DHF and DSS are associated with vascular leakage and neurological sequelae. Our hypothesis was that altered astrocyte activation and morphology would alter the dynamics of the extracellular space and hence, neuronal and vascular function. We investigated the mechanisms of neuropathogenesis DENV infection in rhesus macaques. There were decreased numbers of GFAP immunopositive astrocytes per unit area, although those that remained had increased arbor length and complexity. This was combined with structural hypertrophy of white matter astrocytes in the absence of increased vascular leakage. Combined, these studies show how even low-grade infection with DENV induces measurable changes within the parenchyma of infected individuals.
\end{abstract}

Keywords Flavivirus · Neuropathogenesis · Astrocyte · Glia $\cdot$ Hypertrophy

Andrew G. MacLean

amaclean@tulane.edu

1 Program in Biomedical Science, Tulane Medical School, New Orleans, LA, USA

2 Tulane National Primate Research Center, Covington, LA, USA

3 Department of Biomedical Engineering, Covington, LA, USA

4 Department of Microbiology and Immunology, Tulane Medical School, New Orleans, LA, USA

\section{Introduction}

Arboviruses infect over 100 million humans worldwide annually, with 3 billion at risk for infection. This family of viruses includes Chikungunya, Zika, and dengue (DENV), the last two of which are flaviviruses. The increased risk for infection may be due, in part, to the spread of the mosquito vectors of DENV, Aedes aegypti and Aedes albopictus (WHO 2010).

The traditional focus on neurons has broadened to include astrocytes, microglia, and other cell types when examining neuropathogenesis. Astrocyte activation is the fastest response in the brain to viral infection and often the only change observed (Zlotnik 1968). We have recently observed dramatic changes in astrocyte microanatomy in animals infected with the togavirus Chikungunya (Inglis et al. 2015), the lentivirus simian immunodeficiency virus (SIV) (Lee et al. 2014), or the bacterium Brucella melitensis (Lee et al. 2013a). We have also shown that the order of sequential activation has dramatic alterations in the physiology of astrocytes (Renner et al. 2013). Primates have six to seven subpopulations of astrocytes (Oberheim et al. 2009) compared with two types in rodents, making the nonhuman primate the ideal model for neuropathological examination. Recent evidence suggests that there is specific activation of astrocytes in different areas of brain depending on disease state (Lee et al. 2013b; Torres-Platas et al. 2011). As astrocytes are long established as sentinels for neuroinflammation (Zlotnik 1968), it would be expected that astrocytes would be activated following infection with DENV. Further, astrocytes are known to remain in a phenotype associated with activation after virus is cleared from brain tissues (Lee et al. 2014; Zlotnik 1968), indicating long-term consequences of viral infection.

A common sequela to flavivirus infection, including infection with DENV, is distorted capillaries (Cassetti et al. 2010; Talavera et al. 2004; Velandia-Romero et al. 2012; Zlotnik 
1968; Zompi and Harris 2012), with downstream petechia, and hematomas (Onlamoon et al. 2010). The mechanisms of these are, however, poorly understood. Previous studies at Tulane National Primate Research Center (TNPRC) examined intravenous or subcutaneous inoculation of DENV to establish a model for DENV hemorrhagic fever, which has been associated with neurological sequelae (Maximova et al. 2008; Ramos et al. 1998). We have recently shown that in macaques with active lentiviral infection of brain, there is astrogliosis (Lee et al. 2014) and increased vascular leakage, as evidenced by increased parenchymal fibrinogen (Renner et al. 2012).

Our hypothesis was that DENV infection would induce vascular leakage and changes in the microanatomy of primate astrocytes associated with neuropathology. To address this question, we examined archived brain samples from macaques infected with DENV serotype 2. Our approach was to stain $6-\mu \mathrm{m}$ paraffin sections for fibrinogen, ZO-1, and glial fibrillary acidic protein (GFAP). During the acute phase of DENV infection, we noted that cortical white matter astrocytes were significantly hypertrophied combined with increased complexity. Therefore, in the absence of apparent neuroinflammation or vascular leakage, there was still immune activation of astrocytes and quantifiable changes to their microarchitecture.

\section{Materials and methods}

\section{Ethics statement, animal housing, and selection of tissues}

As has been described previously (Lee et al. 2015), animals were maintained in Animal Biosafety Level 2 housing with a 12:12-h light:dark cycle, relative humidity 30 to $70 \%$, and a temperature of 17.8 to $28.9^{\circ} \mathrm{C}$, as is routine at Tulane National Primate Research Center. TNPRC is fully accredited by the Association for the Assessment and Accreditation of Laboratory Animal Care-International (Animal Welfare Assurance no. A4499-01). All animal-related protocols were overseen by veterinarians and their staff, having been approved by the Institutional Animal Care and Use Committee (IACUC), and in accordance with to the guidelines prescribed by the NIH Guide to Laboratory Animal Care.

Six rhesus macaques were infected by either subcutaneous or intravenous route with DENV serotype 2. Two animals were euthanized for tissue collection at days 2 and 4 postinfection (Table 1). The remaining four animals received DENV twice, separated by 2 months. They were humanely euthanized by the veterinary staff at the TNPRC 26 days after the second inoculation.

Tissues were selected solely on their availability in the TNPRC tissue archive. All of the animals were naïve as regards infectious or pharmacological studies. Frontal cortical tissue from 4 control and 6 DENV virus-infected Chinese- origin rhesus macaques (Macaca mulatta) were used for this study, for a total of 10 animals (Table 1).

\section{Histopathology}

We examined 6- $\mu \mathrm{m}$ paraffin-embedded brain sections stained with hematoxylin and eosin. Inflammatory foci were calculated at low power as previously described (Renner et al. 2012). The area of each section was determined by point counting to determine the total area of the section with a 3.3-mm overlay grid (Howard and Reed 1998). Lesions per square millimeter for each brain section and then animal were calculated and used in graphical comparisons and statistical tests. Other major tissues and organs were evaluated for inflammatory lesion intensity on a scale of 0 to 4 and frequency among the groups of animals.

\section{Immunohistochemistry}

Six-micrometer paraffin sections were incubated with GFAP primary antibody (1:400 dilution, GA-5, Sigma) or fibrinogen (1:100 dilution, Dako) overnight at $4{ }^{\circ} \mathrm{C}$. Following washes with PBS with $0.2 \%$ bovine serum albumin (PBS/BSA; Santa Cruz), they were incubated with secondary antibodies directly conjugated with Alexa 488 (green) or Alexa 568 (red) (Molecular Probes/Invitrogen, Carlsbad, CA) and coverslipped with Prolong Gold with DAPI (Molecular Probes/ Invitrogen). Stained slides were imaged fluorescence microscope (Nikon Eclipse TE2000-U) or confocal (Leica TCS2; Fig. 2 only).

\section{Quantification of astrocyte morphology}

Nonoverlapping fields were imaged at $\times 40$ objective (Nikon Eclipse TE2000-U) and imported into Neurolucida software (MBF Bioscience), as described previously (Inglis et al. 2015). Astrocytes from layers 3-5 were selected randomly for our analysis. An average of 10 astrocytes with clear cell bodies and processes that did not touch the edges of fields in both gray and white matter were chosen from each animal for $2 \mathrm{D}$ reconstruction, importing into and subsequent analyses with Neurolucida Explorer (MBF Bioscience). Morphological measures of cell area, branching points, arbor volume, and length were then generated.

\section{Statistical analyses}

Statistical analyses were performed using GraphPad Prism (version 5, GraphPad Software, La Jolla, CA). Normality was assessed by Kolmogorov-Smirnov test, and data that passed normality were analyzed by unpaired $t$ test. Data that were not distributed normally were assessed by MannWhitney test to determine significance between groups. 
Table 1 Histological examination of tissues

\begin{tabular}{|c|c|c|c|c|c|c|c|}
\hline Animal no. & $\begin{array}{l}\text { Number of } \\
\text { dengue inoculations }\end{array}$ & Inoculation route & Days postinoculation & Age & Fever & Hemorrhage & Other pathologic findings \\
\hline GT46 & 1 & Iv & 2 days & 6.35 & & & Liver lesions \\
\hline GT47 & 2 & $\mathrm{Sq}$ & 2 months +26 days & 6.53 & 102.9 & Petechia & Mild lymphoid hyperplasia \\
\hline GT48 & 2 & Iv & 2 months +26 days & 6.29 & 102.4 & & Lymphoid hyperplasia \\
\hline GT49 & 2 & Iv & 2 months +26 days & 6.4 & 103.5 & Ecchymosis & $\begin{array}{l}\text { Lymphoplasmocytic infiltrates } \\
\text { in multiple organs. } \\
\text { Myocardial degeneration }\end{array}$ \\
\hline GT51 & 2 & $\mathrm{Sq}$ & 2 months +26 days & 6.38 & 103.4 & & $\begin{array}{l}\text { Lymphoplasmocytic infiltrates } \\
\text { in multiple organs }\end{array}$ \\
\hline GT53 & 1 & Iv & 4 days & 6.2 & & & No significant lesions \\
\hline IR52 & 0 & & & 4.53 & & & Colitis \\
\hline EB45 & 0 & & & 7.52 & & & Colitis. Campylobacter pylori \\
\hline FJ77 & 0 & & & 4.8 & & & Colitis \\
\hline GC52 & 0 & & & 3.69 & & & Amyloidosis \\
\hline
\end{tabular}

Results are expressed as mean \pm SEM. For all analyses, significance was set at $p<0.05$.

\section{Determination of cerebral vascular leakage}

Images of slides stained for ZO-1 and fibrinogen expression were captured by fluorescence microscope and analyzed using ImageJ (version 1.43r, NIH). Mean intensity of fibrinogen expression was calculated from single-channel images, as previously described (Renner et al. 2012). Statistics were performed using Prism as above.

\section{Results}

\section{Summary of pathological findings}

Histological examination of tissues from the two acutely infected animals (GT46 and GT53) revealed no lesions with the exception of in liver of GT46 (48 h postinfection) consistent with recent viral infection. The remaining four infected animals (see table for more details) had minimal to mild lymphoid hyperplasia in lymph nodes. Only one animal (GT49) had unusual observation (mild myocardial degeneration), potentially consistent with more severe dengue disease. All four of these animals had low- to middle-grade fever 10 days after the second infection with DENV (ranging from 102.4 to $103.5^{\circ} \mathrm{F}$; normal macaque body temperature is about $\left.101^{\circ} \mathrm{F}\right)$. Two of the four animals receiving the second round of DENV infection had evidence of vascular leakage (GT47 and GT49).

The four control animals were euthanized for wasting or colitis. There was generally minimal inflammation in the colon of these animals and no other significant inflammatory findings.

\section{Infection with serotype 2 dengue decreases the number of GFAP+ astrocytes}

Based on previous studies by ourselves and others, we postulated that DENV infection would alter the numbers of astrocytes per unit area and that these astrocytes would be activated (Inglis et al. 2015; Lee et al. 2013a, b; Snook et al. 2013). To address his question, we stained $6-\mu \mathrm{m}$ paraffin sections with GFAP antibodies as is routine in this lab (Inglis et al. 2015; Lee et al. 2013a, b; Robillard et al. 2016; Snook et al. 2013). To determine if DENV infection affected the density of astrocytes in macaque frontal lobe, the number of GFAP-positive cells per unit area was calculated. Macaques infected with DENV had decreased numbers of white matter astrocytes per unit area compared with controls (from 402 GFAP+ cells $/ \mathrm{mm}^{2}$ to 291 cells $/ \mathrm{mm}^{2}, p<0.0001$; Fig. 1a). Due to the low expression of GFAP-positive cells in cortical gray matter of Chinese rhesus macaques, GFAP-labeled astrocytes could not be evaluated quantitatively (not shown). This was confirmed by quantifying the expression of GFAP using unbiased particle analysis (ImageJ, v1.48u4), as described recently (Inglis et al. 2015). In brief, five images per stained section were captured at $\times 20$ objective. The relative optical density was reduced by $23 \%$ ( $p<0.0001$; Figure $1 \mathrm{~b})$. Thus, DENV serotype 2 infection led to decreased numbers of GFAP immunopositive astrocytes in white matter of macaques.

\section{Dengue virus infection induces process extension}

Astrogliosis is associated with decreased synaptic transmission (Freria et al. 2012). Therefore, as astrocytes form a 


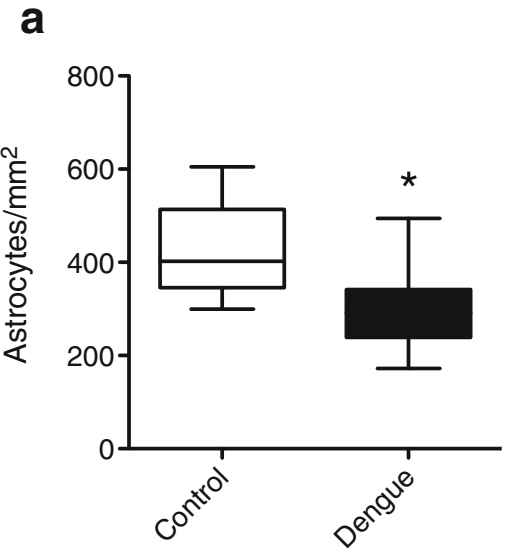

Fig. 1 Decreased GFAP+ astrocytes in white matter following intravenous DENV infection. DENV infection of Chinese rhesus macaques induced a significant decrease in the number of GFAP immunopositive cells in white matter of parietal lobe (a). The total

critical component of the tripartite synapse, we hypothesized that the astrocyte activation would be coupled with altered astrocyte morphology. To quantify these changes, we imported astrocyte images into Neurolucida and analyzed using Neurolucida Explorer. Representative confocal images of astrocytes from control (Fig. 2a) and DENV-infected (Fig. 2b) macaques demonstrate numerous changes.

\section{Morphometric analyses indicate swelling of cell bodies and longer, more highly branched processes in white matter astrocytes}

To determine how astrocyte activation translated into altered overall morphology, fluorescent images of GFAP immunopositive cells were imported into Neurolucida as is routine (Inglis et al. 2015; Lee et al. 2013a, b; Renner et al. 2013; Robillard et al. 2016; Snook et al. 2013). The morphometrics of the cells were then calculated to determine if DENV infection induced an atrophy or hypertrophy. These data are presented in Fig. 3. The cumulative length of astrocyte processes, beginning with cell bodies to the end of processes, was calculated for each astrocyte in gray (Fig. 3a) and white matter (Fig. 3b). While there was no significant change in gray matter, the cell arbor was increased by approximately $50 \%$ in white matter astrocytes following DENV infection (DENV $506 \pm 26 \mu \mathrm{m}^{2}$ vs control $354 \pm 15 \mu^{2}, p<0.0001$ ).

Using the diameter of the astrocyte processes, frusta were created in Neurolucida to determine the arbor volume of the astrocyte processes. There was no significant alteration in volume of astrocyte processes in gray matter (Fig. 3c). However, the arbor volume of the astrocyte processes was increased significantly in white matter astrocytes of animals infected with DENV compared with control brains (control $431 \mu^{3}$ vs DENV $633 \mu \mathrm{m}^{3}, p=0.0018$; Fig. 3d).

\section{b}

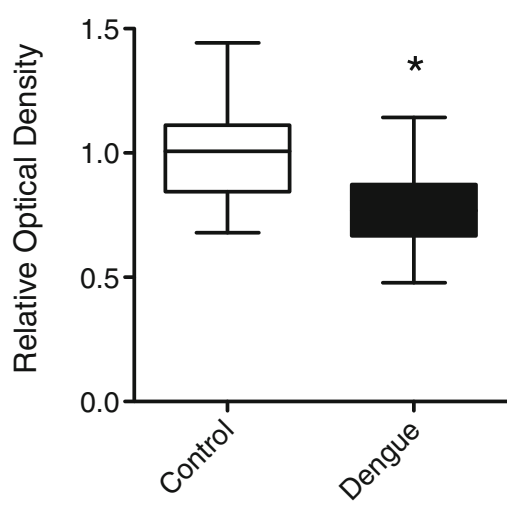

amount of GFAP staining was graphed and analyzed by two-tailed $t$ test. Astrocytes in white matter of DENV-infected macaques had $23 \%$ less GFAP compared with control macaques (b). GFAP staining in gray matter was not suitable for quantitative analyses

\section{Dengue infection induces astrocyte cell body hypertrophy}

As other infections (Chikungunya, SIV, Brucella) have been shown to alter the astrocyte cell body, we assessed cell body atrophy or hypertrophy by measuring the area of the cell body of astrocytes. The cell bodies in control gray matter astrocytes were $133.7 \pm 5.6 \mu \mathrm{m}^{2}$ (Fig. 3e). There was no significant change in cell body area in gray matter astrocytes of DENVinfected macaques $\left(150.5 \pm 6.2 \mu \mathrm{m}^{2}\right)$, However, once again, white matter astrocyte cell bodies were significantly larger following DENV infection in white matter $(189.3 \pm 6.9$ vs $239 \pm 12.7 \mu \mathrm{m}^{2}, p=0.0027$; Fig. 3 f), indicating cytoplasmic expansion/cellular swelling.

\section{Dengue virus induces de novo process formation and increased complexity of white matter astrocytes}

To determine if the increased cell arbor (Fig. 3b) and volume (Fig. 3d) were reflected in an increased number of primary processes, we quantified the number of primary processes. DENV infection did not alter the number of primary processes in gray matter ( $p=0.44$; Fig. $4 a)$. However, the number of primary processes was significantly increased in white matter ( $p=0.008$; Fig. $4 \mathrm{~b}$ ). The complexity of the astrocytes was also assessed by counting the number of nodes and tips per astrocyte. There was no increase in gray matter branching ( $p=0.27$; Fig. $4 \mathrm{c}$ ). The number of bifurcations (nodes) in white matter astrocytes increased significantly in DENVinfected animals $(p<0.0001$; Fig. $4 d)$. As with the other parameters measured, there was no significant increase in the number of astrocyte tips in gray matter following DENV infection ( $p=0.47$; Fig. $4 \mathrm{e})$. There was, however, an increased tip quantity in white matter astrocytes $(p<0.0001$; Fig. 4f) compared with controls. From these studies, we concluded 
Fig. 2 DENV induces hypertrophy of fibrous astrocytes. GFAP immunopositive fibrous astrocytes display distinctive morphology in rhesus macaque parietal lobe (a). There was distinct hypertrophy of astrocytes in macaques previously infected with DENV (b). Images are representative $\mathrm{Z}$-stack composite images captured at $\times 63$
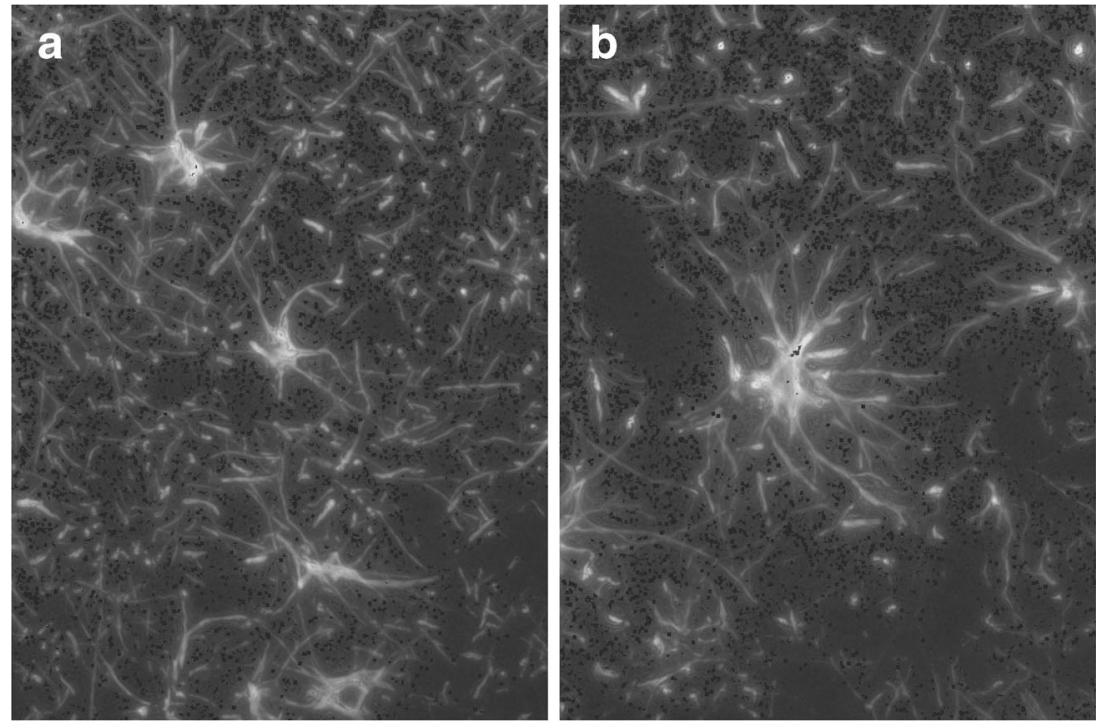

that the increased arbor volume and length are due to extension of the length of individual processes, combined with de novo process formation.

\section{Dengue infection is not correlated with blood-brain barrier disruption}

Finally, we sought to determine if DENV infection with a single serotype induced leakage of serum proteins through the blood-brain barrier and into the parenchyma. To this end, we performed multilabel confocal microscopy to determine if DENV altered ZO-1 expression and plasma fibrinogen infiltration into the parenchyma. We and others have used parenchymal fibrinogen to demonstrate viral-associated blood-brain barrier (BBB) disruption (Luabeya et al. 2000; Renner et al. 2012). In either control macaques, or macaques infected with DENV, we detected minimal fibrinogen (green) in brain parenchyma (Fig. 5a, 5b). Following a similar quantitation method to that used for GFAP expression, there was approximately $22 \%$ increased fibrinogen staining (Fig. 5c); however, this did not reach statistical significance $(p=0.0637)$.

In summary, we observed decreased density of white matter astrocytes in macaques infected with DENV. This was combined with morphologic changes in white matter astrocytes with increase in process length (cell arbor and radius). Furthermore, astrocytes in white matter showed an increase in tip quantity and branching, a further indication of increased astrocyte complexity following DENV infection. Thus, white matter astrocytes are activated in several parameters following DENV infection. That there were no changes in gray matter may reflect the intact $\mathrm{BBB}$, as evidenced by lack of significantly increased parenchymal fibrinogen deposition.

\section{Discussion}

At the time of necropsy (26 days after receiving the second inoculation with DENV), there were no apparent histological changes in the brains of the macaques infected with DENV. However, other organs including multiple lymph nodes were noted to have lymphocytic hyperplasia (not shown). Thus, although DENV did not induce obvious changes in brain, there was limited inflammation in other organ systems in DENV-infected macaques including heart, kidney, and tonsils (not shown).

This study was undertaken to quantify astrogliosis in primates infected with DENV serotype 2. As the role of glial cells in viral neuropathogenesis is becoming increasingly apparent (Inglis et al. 2015; Lee et al. 2014; Velandia-Romero et al. 2012), it was important to quantify the activation of astrocytes with regard to astrocyte proliferation/migration and astrocyte morphology and BBB disruption.

Viral-associated astrogliosis and hypertrophy were noted to occur only in white matter, perhaps an indication of local immune activation (Torres-Platas et al. 2011). That each parameter tested was significantly upregulated in white matter astrocytes regardless of hemorrhagic status would require additional animal studies. It is possibly a result of the long-term nature of astrocyte activation following flaviviral (Zlotnik 1968) or lentiviral (Lee et al. 2014) infection. It is also possible that the nonsignificant elevation of fibrinogen (Fig. 5) reflects that vascular leakage had occurred, but this had recovered by the time of necropsy.

The decreased astrocyte density in white matter is suggestive of either cell migration out of the tissues, downregulation of GFAP, or possibly cell death. It is interesting that West Nile virus, another flaviviral infection, has been correlated with increased glutamate excitotoxicity (Clarke et al. 2014b), 
Fig. 3 Infection with DENV induces hypertrophy of white matter astrocytes. Analyses of the overall morphology of astrocytes revealed subtle changes. There was no significant change in the arbor length (a), volume (c), or cell body area (e) of gray matter astrocytes. However, white matter astrocytes in macaques infected with serotype 2 DENV had significantly increased arbor length (b), volume (d), and cell body area (f)

\section{Grey Matter}
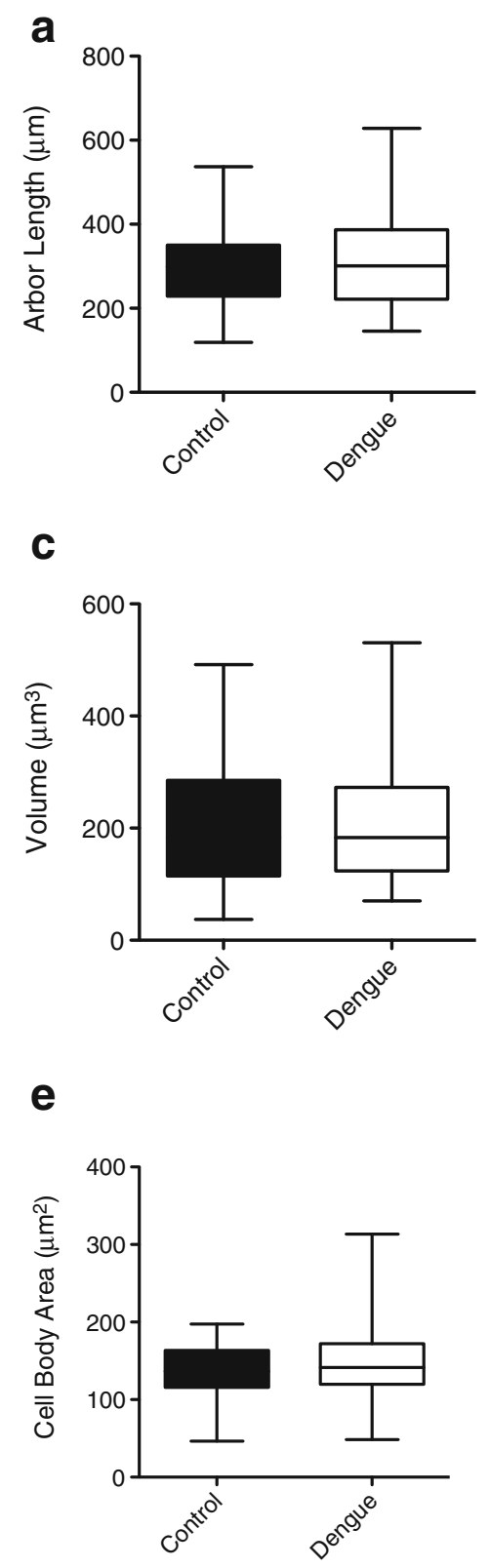

White Matter

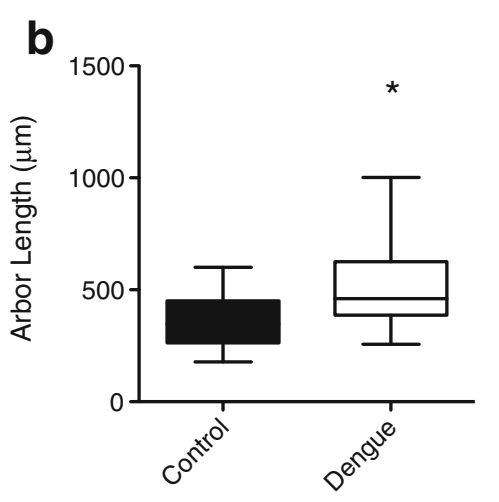

d

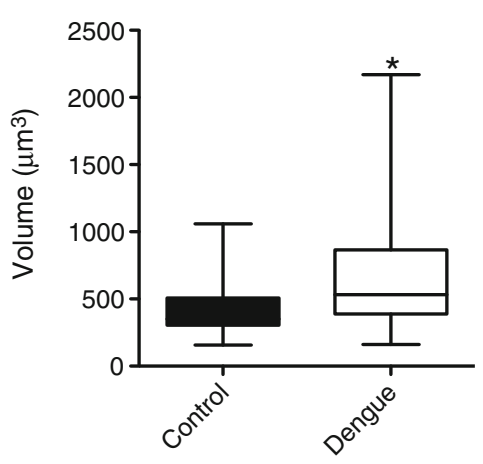

f

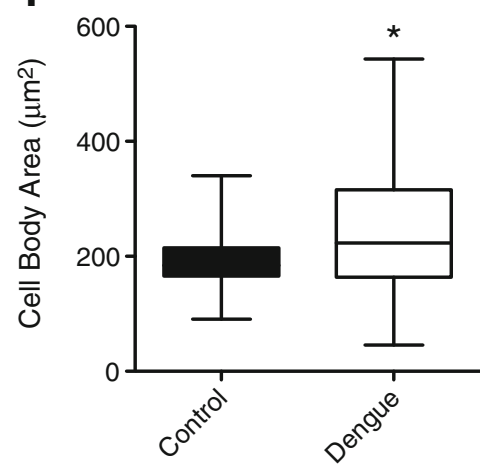

possibly through glial activation (Clarke et al. 2014a). Combined, these data confirm a role for astrocytes in immune activation within brain following flaviviral infection.

We have previously demonstrated morphometric changes in astrocyte processes in response to bacterial infection ( $B$. melitensis; Lee et al. 2013a), togavirus infection (Inglis et al. 2015), or lentiviral infection (Lee et al. 2014) and aberrant behavior (Lee et al. 2015, b). The hypertrophy observed here in white matter was distinct from any of these, with significantly increased cell bodies and numbers of primary processes.

Cytokine secretion and altered adhesion of astrocytes are linked to morphological changes (Renner et al. 2013). It is intriguing that there was a decrease in the number of white matter astrocytes at the same time as an increase in size of astrocytes observed. As white matter fibrous astrocytes are 
Fig. 4 Increased complexity of white matter astrocytes following intravenous infection with DENV. As with the measures of overall morphology, there were no significant changes in the number of primary processes (a), nodes (c), nor tip quantity (e) in gray matter astrocytes. Following infection with DENV, there was a significant increase in the number of primary dendrites (b), nodes/ bifurcations of dendrites (d), and terminal branches/tips (f) in white matter astrocytes

\section{Grey Matter}
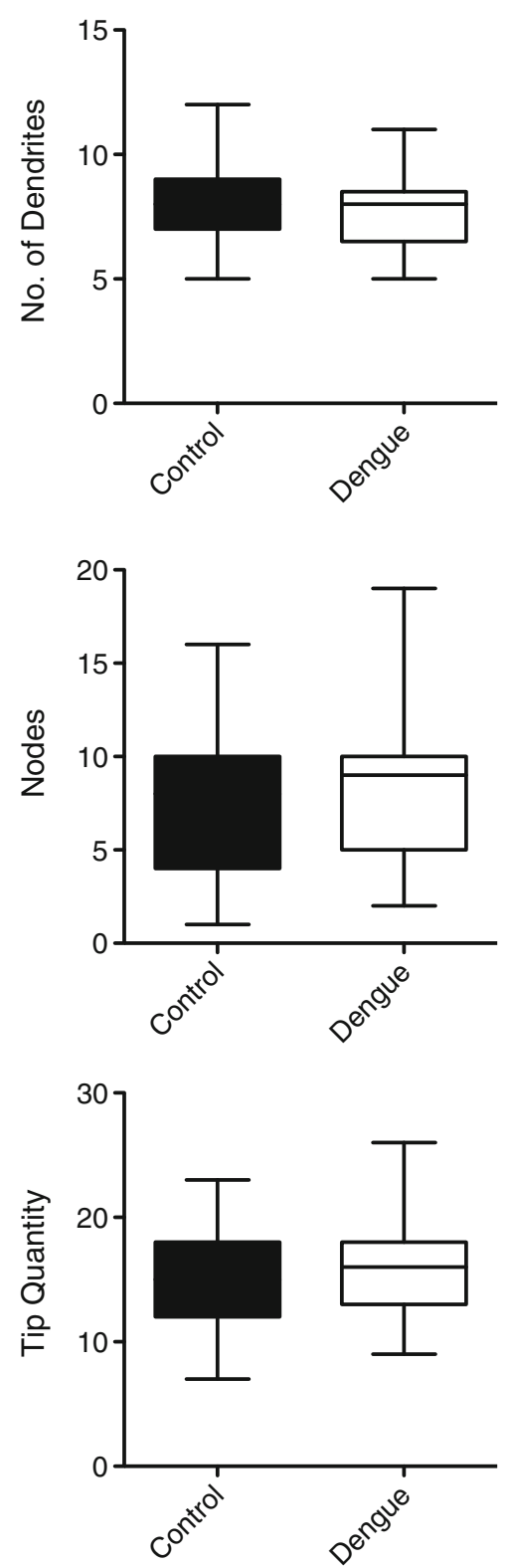
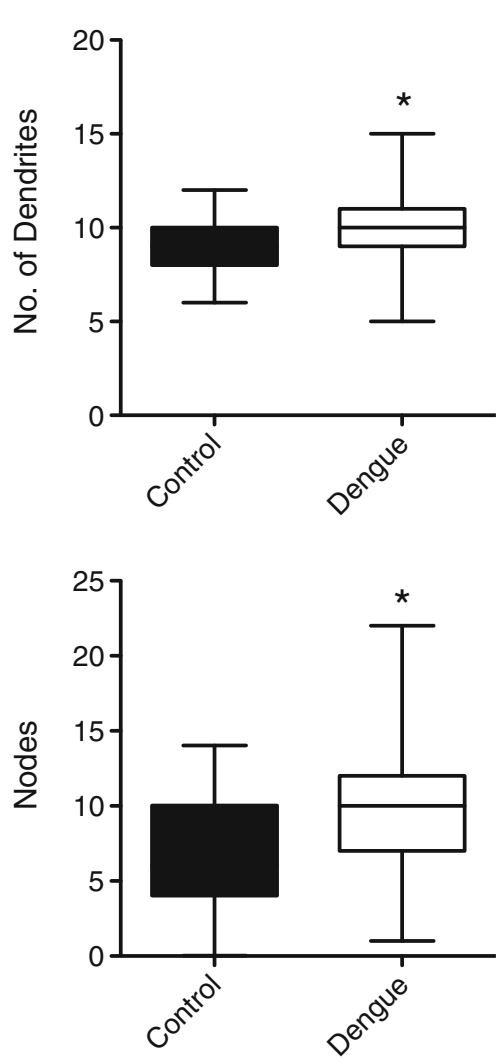

White Matter

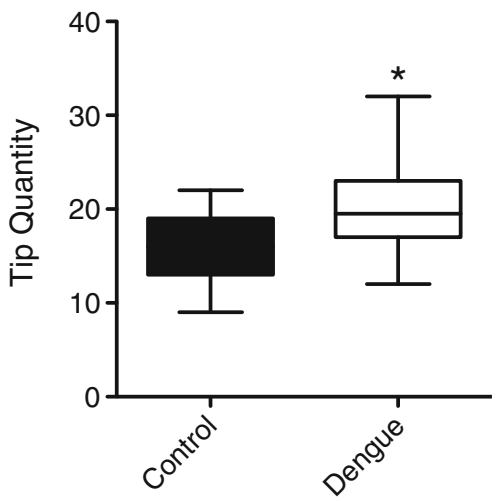

generally evenly spaced out (Oberheim et al. 2009), it is, of course, possible that the increase in size reflects "space filling" by the remaining astrocytes.

Indian rhesus macaques infected with another arbovirus, Chikungunya, have altered astrocyte morphology combined with increased TLR2 expression (Inglis et al. 2015). However, in those animals, while the white matter astrocytes displayed cell body hypertrophy, there were no other significant changes in white matter astrocytes. In addition, the gray matter astro- cyte processes were significantly less complex than in control animals. DENV infection induced morphometric changes in white matter astrocytes similar to those reported in macaques infected with B. melitensis (Lee et al. 2013a) or in depressed humans (Torres-Platas et al. 2011). Previous studies using DENV infection in rodents (Zlotnik 1968) showed acute degeneration of astrocyte processes, although this was observed at 6-8 days after a single round of intracerebral infection, rather than 26 days after a second intravenous or subcutaneous 

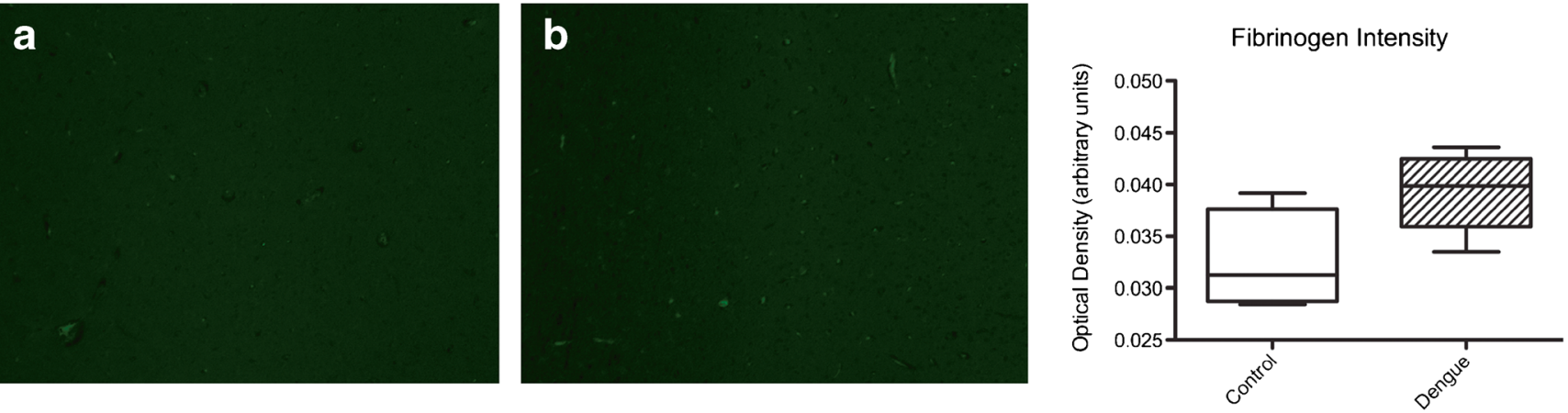

\section{DENGUE}
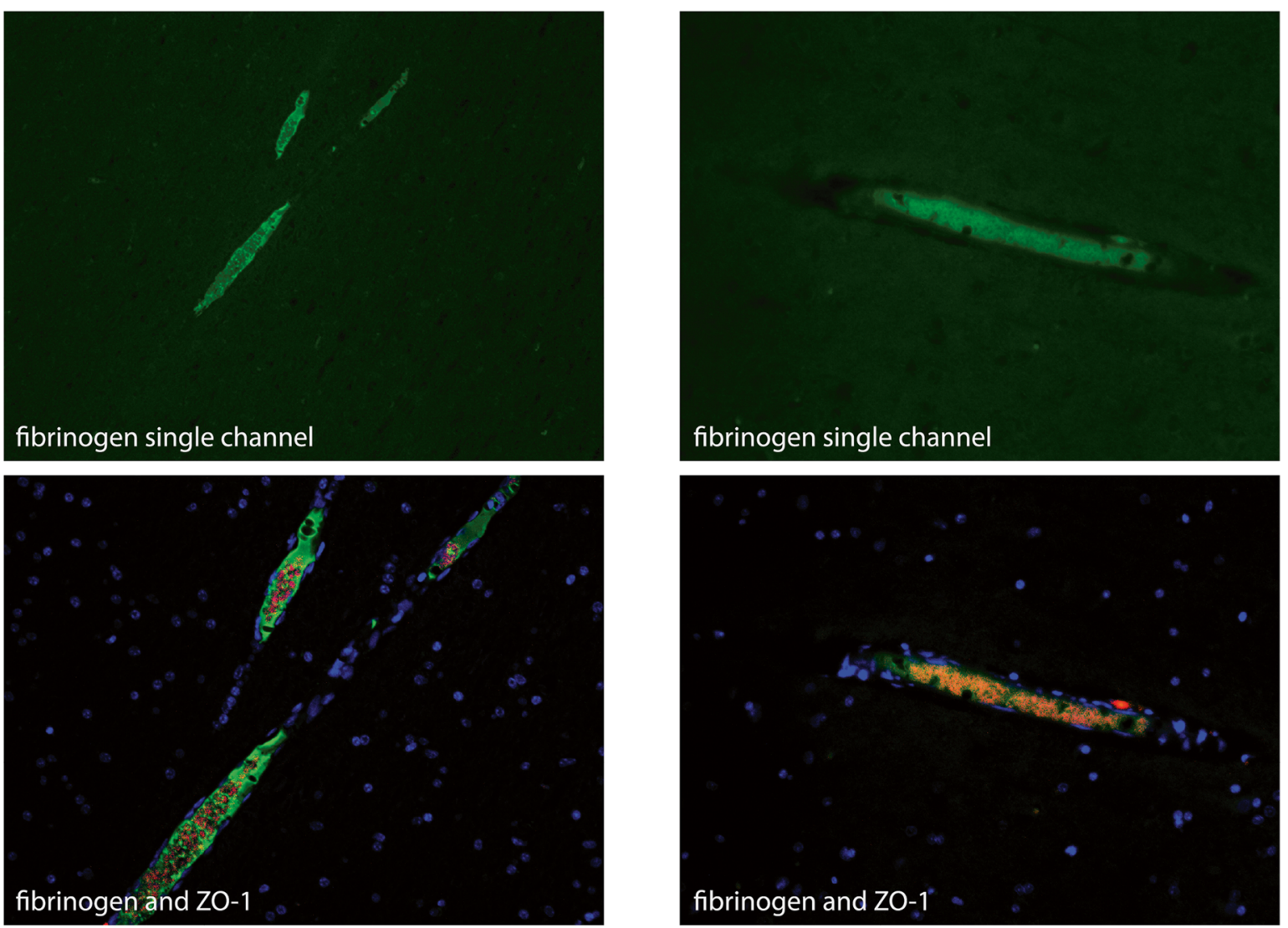

Fig. 5 Cerebrovascular integrity following DENV infection. There was very limited evidence of cerebrovascular leakage in either control (a) or DENV-infected macaques (b). Following unbiased quantitation of

fibrinogen within brain sections, there was $22 \%$ increased fibrinogen staining in DENV animals compared to controls, although this was not significant $(p=0.0637)$

inoculation in this study. However, similar hypertrophy to that observed here was noted in mouse hippocampus and cortex 21 days postinfection with Langat virus (also a flavivirus). Thus, while the astrogliosis observed could conceivably induced by a generalized immune activation observed in the animals infected with DENV (Table 1), we believe this to be unlikely. Unlike in our previous studies using Indian-origin rhesus macaques (Lee et al. 2013a, b; Snook et al. 2013), the control Chinese macaques were euthanized due to inflammatory illness (colitis, amyloidosis).

With the emergence of other flaviviral infections, notably Zika, it will be important to examine if putative vaccine 
candidates or medications inhibit the astrocyte activation. Further, does the glial activation return to a more normal phenotype following resolution of the infection, or is the astrocyte activation more long-lived, even in the absence of virus in brain, as we have recently shown with SIV infection (Lee et al. 2014).

Acknowledgments We thank the pathology faculty and staff at Tulane National Primate Research Center for expertly collecting and archiving samples. This work was supported in part through grants to Dr. MacLean from Tulane University School of Medicine and the Tulane Program in Neuroscience. Ms. Lee was the inaugural TNPRC postgraduate research fellow. PHS grant OD11104, formerly RR00164, was essential for animal husbandry and archiving the tissues for 50 years.

\section{Compliance with ethical standards}

Conflict of interest The authors declare that they have no conflict of interest.

\section{References}

Cassetti MC, Durbin A, Harris E, Rico-Hesse R, Roehrig J, Rothman A, Whitehead S, Natarajan R, Laughlin C (2010) Report of an NIAID workshop on dengue animal models. Vaccine 28:4229-4234

Clarke P, Leser JS, Bowen RA, Tyler KL (2014a) Virus-induced transcriptional changes in the brain include the differential expression of genes associated with interferon, apoptosis, interleukin 17 receptor $\mathrm{a}$, and glutamate signaling as well as flavivirus-specific upregulation of tRNA synthetases. mBio 5:e00902-e00914

Clarke P, Leser JS, Quick ED, Dionne KR, Beckham JD, Tyler KL (2014b) Death receptor-mediated apoptotic signaling is activated in the brain following infection with West Nile virus in the absence of a peripheral immune response. J Virol 88:1080-1089

Freria CM, Velloso LA, Oliveira AL (2012) Opposing effects of toll-like receptors 2 and 4 on synaptic stability in the spinal cord after peripheral nerve injury. J Neuroinflammation 9:240

Howard V, Reed MG (1998) Unbiased stereology: three-dimensional measurement in microscopy. Springer, New York

Inglis FM, Lee KM, Chiu KB, Purcell OM, Didier PJ, Russell-Lodrigue K, Weaver SC, Roy CJ, MacLean AG (2015) Neuropathogenesis of Chikungunya infection: astrogliosis and innate immune activation. $\mathrm{J}$ Neurovirol 22:140-148

Lee KM, Chiu KB, Didier PJ, Baker KC, MacLean AG (2015) Naltrexone treatment reverses astrocyte atrophy and immune dysfunction in self-harming macaques. Brain Behav Immun 50:288297

Lee KM, Chiu KB, Renner NA, Sansing HA, Didier PJ, MacLean AG (2014) Form follows function: astrocyte morphology and immune dysfunction in SIV neuroAIDS. J Neurovirol 20:474-484

Lee, K.M., Chiu, K.B., Sansing, H.A., Didier, P.J., Ficht, T.A., ArenasGamboa, A.M., Roy, C.J., Maclean, A.G., 2013a. Aerosol-induced brucellosis increases TLR-2 expression and increased complexity in the microanatomy of astroglia in rhesus macaques. Frontiers in cellular and infection microbiology 3, 86 .

Lee KM, Chiu KB, Sansing HA, Inglis FM, Baker KC, Maclean AG (2013b) Astrocyte atrophy and immune dysfunction in selfharming macaques. PLoS One 8:e69980

Luabeya MK, Dallasta LM, Achim CL, Pauza CD, Hamilton RL (2000) Blood-brain barrier disruption in simian immunodeficiency virus encephalitis. Neuropathol Appl Neurobiol 26:454-462

Maximova OA, Ward JM, Asher DM, St Claire M, Finneyfrock BW, Speicher JM, Murphy BR, Pletnev AG (2008) Comparative neuropathogenesis and neurovirulence of attenuated flaviviruses in nonhuman primates. J Virol 82:5255-5268

Oberheim NA, Takano T, Han X, He W, Lin JH, Wang F, Xu Q, Wyatt JD, Pilcher W, Ojemann JG, Ransom BR, Goldman SA, Nedergaard M (2009) Uniquely hominid features of adult human astrocytes. J Neurosci Off J Soc Neurosci 29:3276-3287

Onlamoon N, Noisakran S, Hsiao HM, Duncan A, Villinger F, Ansari AA, Perng GC (2010) Dengue virus-induced hemorrhage in a nonhuman primate model. Blood 115:1823-1834

Ramos C, Sanchez G, Pando RH, Baquera J, Hernandez D, Mota J, Ramos J, Flores A, Llausas E (1998) Dengue virus in the brain of a fatal case of hemorrhagic dengue fever. J Neurovirol 4:465-468

Renner NA, Redmann RK, Moroney-Rasmussen T, Sansing HA, Aye PP, Didier PJ, Lackner AA, Maclean AG (2012) S100beta as a novel and accessible indicator for the presence of monocyte-driven encephalitis in AIDS. Neuropathol Appl Neurobiol 38:162-174

Renner NA, Sansing HA, Inglis FM, Mehra S, Kaushal D, Lackner AA, Maclean AG (2013) Transient acidification and subsequent proinflammatory cytokine stimulation of astrocytes induce distinct activation phenotypes. J Cell Physiol 228:1284-1294

Robillard, K.N., Lee, K.M., Chiu, K.B., MacLean, A.G., 2016. Glial cell morphological and density changes through the lifespan of rhesus macaques. Brain Behav Immun.

Snook, E.R., Fisher-Perkins, J.M., Sansing, H.A., Lee, K.M., Alvarez, X., Maclean, A.G., Peterson, K.E., Lackner, A.A., Bunnell, B.A., 2013. Innate immune activation in the pathogenesis of a murine model of globoid cell leukodystrophy. Am J Pathol.

Talavera D, Castillo AM, Dominguez MC, Gutierrez AE, Meza I (2004) IL8 release, tight junction and cytoskeleton dynamic reorganization conducive to permeability increase are induced by dengue virus infection of microvascular endothelial monolayers. J Gen Virol 85: $1801-1813$

Torres-Platas SG, Hercher C, Davoli MA, Maussion G, Labonte B, Turecki G, Mechawar N (2011) Astrocytic hypertrophy in anterior cingulate white matter of depressed suicides. Neuropsychopharmacology 36:2650-2658

Velandia-Romero ML, Acosta-Losada O, Castellanos JE (2012) In vivo infection by a neuroinvasive neurovirulent dengue virus. J Neurovirol 18:374-387

WHO (2010) Working to overcome the global impact of neglected tropical diseases. In: Crompton DWT (ed) WHO press. Switzerland, Geneva

Zlotnik I (1968) The reaction of astrocytes to acute virus infections of the central nervous system. Br J Exp Pathol 49:555-564

Zompi S, Harris E (2012) Animal models of dengue virus infection. Viruses 4:62-82 